\title{
Tailoring photosensitive ROS for advanced photodynamic therapy
}

\author{
Duc Loc Sai ${ }^{1}$, Jieun Lee ${ }^{1}$, Duc Long Nguyen ${ }^{1}$ and Young-Pil Kim (1) ${ }^{1,2,3,4}$
}

\begin{abstract}
Photodynamic therapy (PDT) has been considered a noninvasive and cost-effective modality for tumor treatment. However, the complexity of tumor microenvironments poses challenges to the implementation of traditional PDT. Here, we review recent advances in PDT to resolve the current problems. Major breakthroughs in PDTs are enabling significant progress in molecular medicine and are interconnected with innovative strategies based on smart bio/ nanomaterials or therapeutic insights. We focus on newly developed PDT strategies designed by tailoring photosensitive reactive oxygen species generation, which include the use of proteinaceous photosensitizers, selfillumination, or oxygen-independent approaches. While these updated PDT platforms are expected to enable major advances in cancer treatment, addressing future challenges related to biosafety and target specificity is discussed throughout as a necessary goal to expand the usefulness of PDT.
\end{abstract}

\section{Introduction}

Since the first demonstration for bladder cancer treatment in $1976^{1}$, photodynamic therapy (PDT) has emerged as a clinically approved, noninvasive therapeutic regimen against a range of cancers and nonmalignant diseases ${ }^{2-4}$. PDT can not only suppress tumor growth, but can also stimulate an acute inflammatory response around locally treated tumors, thus encouraging antitumor immunity by releasing secondary inflammatory mediators ${ }^{5-8}$. Generally, PDT relies on three essential components: a photosensitizer (PS), oxygen, and light. The PS administered to the tumor site is activated by light of a specific wavelength, followed by the generation of cytotoxic reactive oxygen species (ROS) in the presence of oxygen; thus, the ROS generated by the PS is the key mechanism by which PDT leads to localized cell death and tissue devastation. In principle, a light-sensitized (excited) PS can react directly with a suitable substrate (unsaturated lipid,

Correspondence: Young-Pil Kim (ypilkim@hanyang.ac.kr)

'Department of Life Science, Hanyang University, Seoul 04763, Republic of

Korea

${ }^{2}$ Department of HY-KIST Bio-Convergence, Hanyang University, Seoul 04763, Republic of Korea

Full list of author information is available at the end of the article

These authors contributed equally: Duc Loc Sai, Jieun Lee, Duc Long Nguyen protein, or nucleic acid) to produce unstable radicals through proton or electron transfer (type I reaction), leading to oxygenated products in the presence of oxygen, such as a superoxide anion radical $\left(\mathrm{O}_{2}{ }^{-}\right)$, a hydroxyl radical $\left(\mathrm{OH}^{*}\right)$, or hydrogen peroxide $\left(\mathrm{H}_{2} \mathrm{O}_{2}\right)$. In turn, the excited PS can react with molecular oxygen to form singlet oxygen $\left({ }^{1} \mathrm{O}_{2}\right)$ through energy transfer (type II reaction); ${ }^{1} \mathrm{O}_{2}$ is a major cytotoxin in PDT, especially at high oxygen contents ${ }^{9,10}$. Although the ratio between type I and type II reactions depends on the type and concentration of PS, the level of oxygen, and the irradiation degree, the mechanistic details between ROS generation and tumor ablation are not fully understood.

As illustrated in Fig. 1, ROS produced by PDT trigger cell death in different ways. The type of cell death induced by PDT depends on the cell type, the PS type or concentration, the intracellular localization, the light dose, and the oxygen partial pressure. Necrosis is a major cell death modality induced by PDT when a chemical PS is confined to the plasma membrane ${ }^{11}$. Apoptosis can also be induced by PDT through oxidative stress when the PS is located at the plasma membrane ${ }^{12}$, as well as in other cellular organelles, such as the nucleus ${ }^{13}$, mitochondria $^{14,15}$, endoplasmic reticulum ${ }^{16,17}$, and lysosomes ${ }^{18,19}$. 


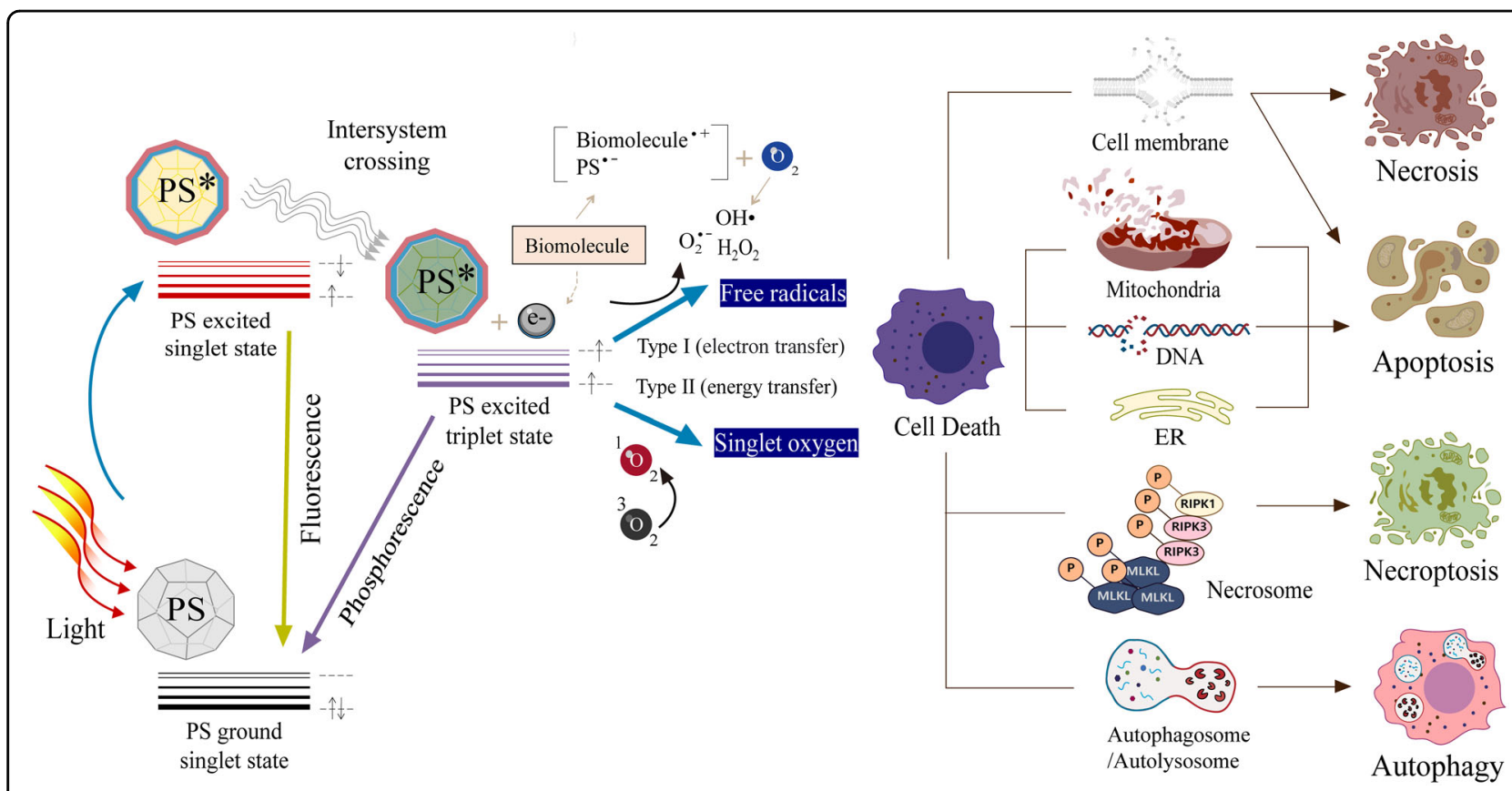

Fig. 1 Schematic illustration of photodynamic reactions (either type I or type II) and cell death pathways in the process of PDT. A PS absorbs energy from light to kill tumor cells via ROS generation. The PDT-induced modes of cell death, including apoptosis, necrosis, necroptosis, and autophagy, depend on the cell type, PS type or concentration, intracellular localization, light dose, and oxygen partial pressure. PDT photodynamic therapy, PS photosensitizer, ER endoplasmic reticulum.

It was reported that PDT led to necroptosis through the formation of necrosomes containing receptor-interacting protein kinase 1 (RIP1) and RIP3 ${ }^{20,21}$. Autophagy is another pathway to cell death by photosensitization, which begins with the formation of spherical autophagosomes that ultimately induce autolysosomes to break down cytoplasmic components ${ }^{22,23}$. The ROS-mediated effects of PDT on cell death have been reviewed in previous reports ${ }^{24-26}$.

Despite the significant progress in the use of PDT in cancer therapy, it still faces certain limitations, including the accumulation or photobleaching of photosensitizers, the limited delivery of light doses, and ineffectiveness in tumor hypoxia ${ }^{27}$. For example, tetrapyrrole drugs (e.g., porphyrin or chlorin) or relatively safe prodrugs (e.g., aminolevulinic acid) often cause sunlight-induced photodermatosis or hepatic porphyria ${ }^{28,29}$. Furthermore, light penetration was limited to $1-2 \mathrm{~mm}$ underneath the skin in a clinical study ${ }^{30}$, and the tumor hypoxia that mainly occurs in the central areas of most solid tumors was reported to be resistant to $\mathrm{PDT}^{31}$. To circumvent these issues, various PDT modalities have been recently developed in combination with smart materials or other therapeutic modalities.

Here, we review recent advances in PDTs to resolve the current problems. To avoid redundancy with general review articles on PDT, as reported previously ${ }^{32-36}$, we focus on innovative PDT strategies based on tailoring photosensitive ROS generation, which include the use of proteinaceous PS, self-illumination, or oxygen-independent methods. To expand the usefulness of PDT, we further discuss ROS generation and targeting models in advanced PDT and summarize recent research results.

\section{PDT with proteinaceous PS}

The anticancer efficiency of PDT largely depends on the improvement of PSs. Much effort has been made to develop PSs as chemical drugs that preferentially accumulate in aberrant vasculature and tumor tissues, but not in normal tissues. The abilities of PSs have evolved through their first (oligomeric hematoporphyrin derivatives), second (pure and synthetic compounds), and third generations (nano- or polymer-conjugates of secondgeneration PSs with targeting moieties) ${ }^{37}$. These chemical drugs or prodrugs rely primarily on localized treatment via a passive targeting route (i.e., enhanced permeability retention (EPR) effects in leaky vasculature and tortuous blood vessels) and/or via low-density lipoprotein receptors overexpressed in tumor cells ${ }^{38}$. Nonetheless, the distribution and ROS generation of PS can vary in vivo, and the heterogeneous accumulation of PS in tumor regions makes it difficult to determine the light dose required to induce photodynamic effects and to avoid photobleaching reactions. Especially since chemical PSs capitalize on high ROS production at high concentrations, this method could lead to nonspecific side reactions or 
Table 1 Photochemical properties of proteinaceous PSs.

\begin{tabular}{|c|c|c|c|c|c|c|}
\hline Name & $\mathrm{Ex} / \mathrm{Em}$ & $\mathrm{MW}(\mathrm{kDa})$ & Chromophore & ROS Type & $Q_{Y}$ & Ref. \\
\hline miniSOG & $448 / 500$ & 14 & FMN & ${ }^{1} \mathrm{O}_{2}$ & 0.47 & 41 \\
\hline SOPP & 439/488(515) & 14 & FMN & ${ }^{1} \mathrm{O}_{2}$ & 0.25 & 43,44 \\
\hline Pp2FbFP ${ }^{L 30 M}$ & $449 / 495$ & 30 & FMN & ${ }^{1} \mathrm{O}_{2}$ & 0.25 & 45 \\
\hline KillerRed & $585 / 610$ & 27 & $Q^{65} Y^{66} G^{67}$ & $\mathrm{O}_{2}^{\cdot-}$ & 0.25 & $46-48$ \\
\hline SuperNova & $579 / 610$ & 29 & $Q^{65} Y^{66} G^{67}$ & ${ }^{1} \mathrm{O}_{2} / \mathrm{O}_{2}^{\cdot-}$ & 0.30 & 49 \\
\hline mKillerOrange & $512 / 555$ & 27 & $Q^{65} W^{66} G^{6}$ & $\mathrm{O}_{2}^{\cdot-}$ & 0.42 & 50 \\
\hline SuperNova Green & $440 / 510$ & 29 & $A^{44} Q^{65} W^{66} G^{67}$ & $\mathrm{O}_{2}^{\cdot--}$ & 0.23 & 51 \\
\hline mKate2 & $588 / 633$ & 26 & $M^{65} Y^{66} G^{6}$ & ${ }^{1} \mathrm{O}_{2} / \mathrm{O}_{2}^{\cdot-}$ & 0.4 & 52 \\
\hline
\end{tabular}

PS photosensitizer, Ex excitation wavelength, Em emission wavelength, MW molecular weight, ROS reactive oxygen species, QY fluorescence quantum yield, Ref. references, FMN flavin mononucleotide, SOG single-oxygen generator, SOPP singlet-oxygen photosensitizing protein.

dark cytotoxicity. To increase water solubility and target specificity while decreasing toxicity, chemical PSs with high hydrophobicity and lipophilicity can be conjugated with protein carriers ${ }^{39}$ or antibodies ${ }^{40}$. However, this still requires an elaborate conjugation process and cannot prevent the side effects of released chemical PSs.

To resolve the innate problems of chemical PSs, a class of flavin-binding proteins (miniSOG, SOPP, and $\mathrm{Pp} 2 \mathrm{FbFP}^{\mathrm{L} 30 \mathrm{M}}$ ) or fluorescence (FL) protein variants (KillerRed, SuperNova, mKate, and KillerOrange) have been identified and proven to have light-activated ROSgenerating properties (Table 1). Among the ROS, two species (superoxide and singlet oxygen) are primarily generated from these proteins. Singlet oxygen is more reactive and damaging in biological systems because more cells lack antioxidant enzymes against singlet oxygen than against superoxide. Indeed, it is noteworthy that protein photoreceptors found in plants and marine invertebrates have been distinctly fine-tuned to adapt for high lightenergy efficiency over their lifetime. As a result of this high energy yield, ROS can be produced in large quantities inside the cell, but organisms have evolved selfdefense mechanisms against internal ROS production by precisely regulating multiple signaling pathways. Proteinaceous PSs are created by engineering photosensitive proteins derived from living organisms that evolved in various environments. A mini singlet-oxygen generator (miniSOG) is a green fluorescent protein variant (106 amino acids) engineered from the LOV2 domain of Arabidopsis thaliana phototropin $2^{41}$. The flavin mononucleotide (FMN) in miniSOG serves as a chromophore, as well as an endogenous cofactor ${ }^{42}$. MiniSOG/FMN, as a photosensitizing protein, was developed to yield higher ${ }^{1} \mathrm{O}_{2}$ than free FMN. This protein was mutated at FMNbinding sites to further increase the ${ }^{1} \mathrm{O}_{2}$ quantum yield, resulting in singlet-oxygen-producing protein (SOPP), SOPP2, and SOPP3 ${ }^{43,44}$. Pp2FbFP ${ }^{\mathrm{L} 30 \mathrm{M}}$ is a site-directed variant (L30M) from a flavin-binding protein, Pp2FbFP, found in Pseudomonas sputita ${ }^{45}$. In contrast to its relatively low-FL quantum yield, it was reported that the generation of ${ }^{1} \mathrm{O}_{2}$ in $\mathrm{Pp}_{2} \mathrm{FbFP}^{\mathrm{L} 30 \mathrm{M}}$ is approximately threefold higher than that of miniSOG ${ }^{45}$. KillerRed was the first genetically encoded red fluorescent protein with high ROS production ${ }^{46}$, whose generation of ROS was reported to be 1,000-fold higher than that of enhanced green fluorescent protein ${ }^{46-48}$. This $26-\mathrm{kDa}$-protein has a barrel shape with 11 antiparallel beta-sheets and a QYG $\left(\mathrm{Glu}^{65}-\mathrm{Tyr}^{66}-\mathrm{Gly}^{67}\right)$ chromophore ${ }^{48}$, and predominantly undergoes type I reactions rather than type II reactions. However, KillerRed tends to form dimers ${ }^{46}$, which may prevent efficient fusion with other proteins of interest in applications. To overcome this limitation, SuperNova was identified in 2013 as a monomeric variant (mutations at six amino acids) of KillerRed ${ }^{49}$. Despite the monomeric PS, SuperNova showed a $5 \%$ increase in ${ }^{1} \mathrm{O}_{2}$ generation and a $10 \%$ reduction in superoxide generation ${ }^{49}$. Monomeric KillerOrange (mKillerOrange) ${ }^{50}$ or SuperNova Green $^{51}$ was further developed by mutating a single amino acid (Y66W) or double amino acids (V44A and Y66W) of SuperNova, enabling blue or green light illumination, respectively. An mKate variant called mKate2, a monomeric far-red fluorescent protein known to generate both superoxide and ${ }^{1} \mathrm{O}_{2}$ was derived from TagRFP ${ }^{52}$.

These photosensitizing proteins have remarkable advantages over chemical PSs. First, upon light illumination, chromophores of proteinaceous PSs are capable of a high rate of intersystem crossing to generate ROS, compared to chemical dyes ${ }^{53}$. Second, they can be designed as genetically encoded proteins that are locally expressed in subcellular organelles with appropriate signal sequences, enabling precisely targeted and spatially controlled photodynamic action at the intracellular level. Third, the photodynamic action of locally expressed proteins has little effect on molecules in other regions, due to the 
ability to control the spatial distribution and the reaction time to light. For example, superoxide anions are known to have a lifetime of $<15 \mathrm{~min}$ and a diffusion distance of $<0.5 \mu \mathrm{m}^{54}$, whereas singlet oxygen has a shorter lifetime of $<0.04 \mu$ s and a more limited effective diffusion distance of $<20 \mathrm{~nm}$ at the site of PS localization in the cell ${ }^{55,56}$. Therefore, with the diminutive lifespan of ROS, it is reasonable that if proteinaceous PDT is precisely regulated at the site of expression, it can perform rapid local treatment without damage to nontarget molecules under a given light and time condition. It is also important to note that proteinaceous PSs have lower phototoxicities than chemical PSs in the dark ${ }^{57}$. Significantly, photodynamic cytotoxicity or tumor devastation has been achieved using either protein-encoding genes transfected into mammalian cells ${ }^{58,59}$ or bacterially expressed proteins ${ }^{60}$. By this principle, proteins bound with proteinaceous PS can functionally be knocked out in living cells, which is known as chromophore-assisted light inactivation, an optogenetic ablation method with high spatiotemporal resolution $^{61,62}$. For the delivery of proteinaceous PS-encoding genes, a tumor-specific delivery system is required, and low-expression genes must be overcome, but this method is expected to facilitate the usability of PDT in basic and applied life science research.

\section{PDT with self-illumination}

Despite recent advances in the field of PSs, the efficient delivery of light energy to PSs is still a challenge because external light sources are limited in tissue permeability and cannot reach tumors deep within the body or behind organs. Even near-infrared light, generated by improved laser technology, cannot easily travel deeper than a few millimeters into a tissue or organ ${ }^{63}$. Most PSs absorb strongly at approximately $400 \mathrm{~nm}$ and weakly at 600 $-800 \mathrm{~nm}^{64}$, which causes a serious drop in the efficiency of ROS generation in vivo. Alternatively, two external light-independent methods, bioluminescence (BL) and chemiluminescence $(\mathrm{CL})$, have been attractive because efficient energy transfer is possible through the induction of internal light near cancer tissues without the need for a high-intensity pulsed light source or expensive lightgenerating devices ${ }^{65}$. The external light-independent applications in PDT are summarized in Table 2.

$\mathrm{CL}$ is the light emitted from a chemical reaction primarily between a peroxide $(\mathrm{O}-\mathrm{O})$-containing molecule and an electron-rich group. The oxidation of luminol is a representative $\mathrm{CL}$ reaction; it occurs in an alkaline solution in the presence of hydrogen peroxide and oxidant catalysts, such as $\mathrm{Fe}^{2+}, \mathrm{Cu}^{2+}, \mathrm{Co}^{2+}$, periodate ions, or hydrogen peroxidase $^{66}$. The oxidation product from luminol emits a strong blue $\mathrm{CL}$ at a maximum of $425 \mathrm{~nm}^{67}$, and is widely used in immunoassays with horseradish peroxidase (HRP)conjugated antibodies $^{68}$. Owing to their simplicity and the effectiveness of their light generation and ${ }^{1} \mathrm{O}_{2}$ production, CL-generating systems have been developed for PDT. An initial report on CL-based PDT was published in 2012 by Wang's group ${ }^{69}$. They used luminol and hydrogen peroxide as CL-generating molecules and cationic oligo( $p$-phenylene vinylene) as a PS. CL without external light irradiation activated PS, killing the cancer cells or pathogenic fungus (Candida albicans) surrounding the complex through ROS generation. A smart material based on CL was described, which used semiconducting polymer dots (Pdots) with an incorporated chemical PS, meta-tetra(hydroxyphenyl)chlorin (m-THPC), which was conjugated with HRP (a CL catalyst) and folic acid (a tumor-targeting ligand $)^{70}$. The $\mathrm{CL}$ induced by luminol $/ \mathrm{H}_{2} \mathrm{O}_{2}$ on the surfaces of Pdots showed increased cytotoxicity in $\mathrm{C} 6$ glioma cells and MCF-7 breast cancer cells that overexpressed folate receptors on their cell membranes. In particular, Pdots have a large Stokes shift at excitation/emission ranging from $420 \mathrm{~nm}$ to $650 \mathrm{~nm}$, leading to highly efficient ROS production via simultaneous CL resonance energy transfer (CRET, between luminol and Pdots) and FL resonance energy transfer (FRET, between Pdots and $\mathrm{m}$-THPC). Similar to Pdots, organic nanoparticles (NPs) were synthesized by coupling electron donors and acceptors inside the NPs to increase FRET efficiency and ROS generation ${ }^{71}$. The mixed organic fluorogens gave rise to aggregation-induced emission in a confined area, thus providing bright $\mathrm{FL}$ and $\mathrm{CL}$, as well as stable ${ }^{1} \mathrm{O}_{2}$ generation. More recently, nanoconjugates consisting of luminol and a transducer (a self-degradable polymer or carbon dot) were reported to be implemented in $\mathrm{PDT}^{72,73}$. They also resulted in the successful inhibition of tumor growth with low background cytotoxicity. However, despite the advantages of $\mathrm{CL}$, most studies rely on luminol, which is a high-energy molecule with potential biotoxicity to living systems. In addition, $\mathrm{H}_{2} \mathrm{O}_{2}$ and a catalyst must be added or present in the microenvironment for chemiexcited ${ }^{1} \mathrm{O}_{2}$ production. When applied directly in clinical use, these nanoconjugates may cause another toxicity issue and require further optimization.

$\mathrm{BL}$ is a subset of $\mathrm{CL}$, in which the light-generating chemical reaction occurs by a process naturally catalyzed by enzymes inside living organisms (e.g., luciferase-luciferin $)^{74,75}$. Therefore, compared to CL, it is anticipated that the photon-emitting capability of BL is more compatible with biological systems. Similar to the advantages observed in proteinaceous PSs, luciferases, as BL-emitting enzymes, can be utilized in the form of either a genetically encoded reporter ${ }^{76}$ or an expressed pure protein ${ }^{77,78}$. Additionally, together with recent advances in detection technology, BL-based assays provide high sensitivity, with levels approaching $10^{-20}$ moles (equivalent to a few molecules per cell) and a large dynamic range $\left(10^{6}\right.$ to $10^{8}$ orders of magnitude). Although BL yields lower light intensities than FL or $\mathrm{CL}$, it is important that a 
Table 2 Representative studies on self-illuminating PDTs.

\begin{tabular}{|c|c|c|c|c|}
\hline $\begin{array}{l}\text { Energy source } \\
\text { (component) }\end{array}$ & Transducer or carrier & Photosensitizer (type) & Targeting strategy (target) & Ref. \\
\hline $\begin{array}{l}\mathrm{CL} \\
\text { (luminol/ } / \mathrm{H}_{2} \mathrm{O}_{2} \text { ) }\end{array}$ & Oligo ( $p$-phenylene vinylene) & $\begin{array}{l}\text { Oligo ( } p \text {-phenylene vinylene) } \\
\text { (Chemical) }\end{array}$ & $\begin{array}{l}\text { Passive targeting } \\
\text { (xenograft mice of HeLa; Fungus, C. albicans) }\end{array}$ & 69 \\
\hline $\begin{array}{l}\mathrm{CL} \\
\text { (luminol/ } / \mathrm{H}_{2} \mathrm{O}_{2} \text { ) }\end{array}$ & Polymer dot & $\begin{array}{l}\text { m-THPC (Foscan) } \\
\text { (Chemical) }\end{array}$ & $\begin{array}{l}\text { Active targeting } \\
\text { (C6 and MCF-7 cells) }\end{array}$ & 70 \\
\hline $\begin{array}{l}\mathrm{CL} \\
\left(\mathrm{CPPO} / \mathrm{H}_{2} \mathrm{O}_{2}\right)\end{array}$ & Pluronic F-127 & $\begin{array}{l}\text { TPE-BT-DC } \\
\text { (Chemical) }\end{array}$ & $\begin{array}{l}\text { Passive targeting } \\
\text { ( } 4 \mathrm{~T} 1 \text { cells and xenograft mouse) }\end{array}$ & 71 \\
\hline $\begin{array}{l}\mathrm{CL} \\
\text { (luminol/ } \mathrm{H}_{2} \mathrm{O}_{2} \text { ) }\end{array}$ & PEG polymer & $\begin{array}{l}\text { Chlorine e6 } \\
\text { (Chemical) }\end{array}$ & $\begin{array}{l}\text { Passive targeting } \\
\text { (macrophage, some cancer cells, and } \\
\text { inflammation mouse) }\end{array}$ & 72 \\
\hline $\begin{array}{l}\mathrm{CL} \\
\text { (luminol/ } / \mathrm{H}_{2} \mathrm{O}_{2} \text { ) }\end{array}$ & Carbon dot & $\begin{array}{l}\text { Chlorine e6 } \\
\text { (Chemical) }\end{array}$ & $\begin{array}{l}\text { Passive targeting } \\
\text { (SMMC-7721 cells and xenograft mouse) }\end{array}$ & 73 \\
\hline $\begin{array}{l}\mathrm{BL} \\
\text { (Fluc/luciferin) }\end{array}$ & Gene transfection agent & $\begin{array}{l}\text { Rose bengal } \\
\text { (Chemical) }\end{array}$ & $\begin{array}{l}\text { Passive targeting } \\
\text { (NIH } 3 T 3 \text { cells) }\end{array}$ & 79 \\
\hline $\begin{array}{l}\mathrm{BL} \\
\text { (Rluc variant/CTZ) }\end{array}$ & Quantum dot & $\begin{array}{l}\text { m-THPC (Foscan) } \\
\text { (Chemical) }\end{array}$ & $\begin{array}{l}\text { Passive targeting } \\
\text { (A549 cells and xenograft mice) }\end{array}$ & 80 \\
\hline $\begin{array}{l}\mathrm{BL} \\
\text { (Rluc variant/CTZ) }\end{array}$ & Quantum dot & $\begin{array}{l}\text { Chlorine e6 } \\
\text { (Chemical) }\end{array}$ & $\begin{array}{l}\text { Passive targeting } \\
\text { (B16F10, CT26, or LLC cells and their xenograft mice) }\end{array}$ & 81 \\
\hline $\begin{array}{l}\mathrm{BL} \\
\text { (Fluc/luciferin) }\end{array}$ & PLGA nanoparticle & $\begin{array}{l}\text { Rose bengal } \\
\text { (Chemical) }\end{array}$ & $\begin{array}{l}\text { Passive targeting } \\
\text { (MCF-7 and HeLa cells) }\end{array}$ & 82 \\
\hline $\begin{array}{l}\text { BL } \\
\text { (Gluc variant/CTZ) }\end{array}$ & Mini-ferritin & $\begin{array}{l}\text { Protoporphyrin IX } \\
\text { (Chemical) }\end{array}$ & $\begin{array}{l}\text { Passive targeting } \\
\text { (SK-BR-3 and MDA-MB-231 cells) }\end{array}$ & 83 \\
\hline $\begin{array}{l}\text { BL } \\
\text { (Nluc) }\end{array}$ & Gene transfection agent & $\begin{array}{l}\text { minisOG } \\
\text { (Protein) }\end{array}$ & $\begin{array}{l}\text { Passive targeting } \\
\text { (SK-BR-3 cells) }\end{array}$ & 84 \\
\hline $\begin{array}{l}\mathrm{BL} \\
\text { (Rluc variant/CTZ) }\end{array}$ & None & $\begin{array}{l}\text { KillerRed, miniSOG } \\
\text { (Protein) }\end{array}$ & $\begin{array}{l}\text { Active targeting } \\
\text { (Six BC cells, patient primary cells, and BC- } \\
\text { xenograft mouse) }\end{array}$ & 60 \\
\hline
\end{tabular}

$C L$ chemiluminescence, $B L$ bioluminescence, $m$-THPC meta-tetra(hydroxyphenyl)-chlorin, $C P P O$ bis[2,4,5-trichloro-6-(pentyloxycarbonyl) phenyl] oxalate, $T P E-B T-D C$ methoxy-substituted tetraphenylethylene (TPE), benzothiadiazole (BT), and dicyanovinyl (DC), PLGA poly(lactide-co-glycolide), Fluc firefly luciferase, Rluc Renilla luciferase, Gluc Gaussia luciferase, Nluc, NanoLuc ${ }^{\circledR}$ luciferase, CTZ coelenterazine, BC breast cancer.

background signal is virtually absent due to the lack of inherent BL reactions in mammalian cells. Therefore, the advantages of BL make it possible to intrinsically regulate ROS production, enabling various PDT approaches. The usability of BL in PDT was initially examined by Theodossiou and coworkers in $2003^{79}$. They used a genetically encoded firefly luciferase (Fluc) as an intracellular BL source. The coaddition of luciferin (a substrate for Fluc) and rose bengal (a photosensitizing natural dye) into Fluctransfected cells significantly diminished cell viability due to intracellular ROS production via BL resonance energy transfer (BRET) between the BL compound and rose bengal. Lai's group ${ }^{80}$ and Yun's group ${ }^{81}$ demonstrated more advanced BL-based PDTs. The core principle was the conjugation of luciferase protein and semiconducting nanocrystals, quantum dots (QDs). This conjugate led to efficient energy transfer from a BL compound into QDs upon the addition of substrate. Consequently, these enzyme-fused QDs can sensitize various PSs at different wavelengths due to the size-tunable property of QDs. The authors reported that this method effectively suppressed tumor growth by BL, which was not affected by tissue depth. They also used brighter, more stable, and/or redshifted proteins, Renilla luciferase (Rluc) variants and their synthetic substrate coelenterazine. In addition to metallic NPs, it was reported that luciferase was able to be fused with polymeric NPs such as poly(lactic-co-glycolic acid $)^{82}$ or naturally occurring protein NPs such as ferri$\operatorname{tin}^{83}$ to increase protein stability and/or ROS production in cells, and their abilities to exert PDT were demonstrated in different types of cancer cells.

While these BL-based PDT strategies were successfully employed in conjunction with traditional chemical PSs, they still encountered inherent problems in vivo (e.g., 
slow degradability or light-sensitive side effects), as mentioned above. To overcome chemical PS toxicity, it was demonstrated that the delivery of genes encoding proteinaceous PS and luciferase was used to kill cancer cells $^{84}$, which would be more beneficial when using tumor-specific promoters. Recently, this idea was further validated using fusion proteins rather than gene expression by Kim's group ${ }^{60}$. They designed fusion proteins consisting of luciferase, proteinaceous PS, and a tumortargeting peptide and demonstrated that the protein probe promoted significant cytotoxicity in cancer cells through the generation of BL-sensitive ROS on the plasma membrane, which is denoted by BL-induced proteinaceous PDT (BLiP-PDT). It is important to note that the extremely weak BL intensity (approximately $0.3 \%$ lower than that of conventional LED light irradiation) induced a relatively large amount of ROS transiently and locally in proximity to the targeted regions, thus resulting in excellent therapeutic effects in both patient-derived primary cells and tumor-bearing mice. In addition, BLiPPDT did not encounter light penetration problems in vivo, thus resulting in a therapeutic platform without an external light source.

Based on these findings, the CL- or BL-based approach has great potential in PDT, especially in terms of its simplicity and independence from external light. Nonetheless, these methods still require more research to be clinically useful, which includes improved tumor selectivity and reduced immunogenicity. For example, with a few exceptions ${ }^{60,70}$, most studies relied on passive targeting via EPR, which may adversely affect normal cells, as observed in classical PDTs. Moreover, the long-term in vivo toxicity of some nanomaterials or $\mathrm{CL}$ (or $\mathrm{BL}$ )inducing chemical substrates (e.g., luminol, luciferin, or coelenterazine) is of special concern in current studies. In this regard, CL- or BL-based PDTs combined with antibody fragments or nonimmunogenic polymers are required to achieve self-illuminating cancer treatment.

\section{PDT with oxygen-independent strategies}

Tumor hypoxia characterized by deficient tissue oxygenation is a major hurdle in PDT because oxygen is a prerequisite for $\mathrm{PDT}^{85}$. Tumor hypoxia can occur in two forms: permanent or transient. In the first form, the rapid progression of the tumor depletes the blood supply, leading to hypoxia ${ }^{86}$. In the second form, PDT itself causes rapid inhibition of the tumor vasculature, leading to rapid depletion of the local oxygen supply ${ }^{87}$. In any case, solid tumors with a high percentage of hypoxic cells have strong resistance to PDT, making it very difficult to continuously perform $\mathrm{PDT}^{88}$. The key molecular mechanism of hypoxia begins with the activation of hypoxia-inducible factor-1 (HIF-1), which is a major effector and transcriptional regulator. Not all PDT treatments induce persistent hypoxia, but it was reported that PDT-mediated oxidative stress (especially very long treatments for $12-14 \mathrm{~h}$ ) led to the activation of HIF- $1 \alpha^{89}$. Indeed, this signal transduction pathway provides an important clue to the solution. For example, Heger's group demonstrated that HIF-1 inhibition not only reduced survival signaling, but also enhanced PDT efficacy, resulting in cancer cell death by PDT even under hypoxic conditions ${ }^{90,91}$.

In addition to the above method, much effort has been made to date to overcome the hypoxia-related problem of PDT. A review article on these strategies was recently published by Yoon's group ${ }^{92}$. They described three main categories for PDT strategies to avoid hypoxia: i) tumor hypoxia-relieving strategies (e.g., delivery of $\mathrm{O}_{2}$ into tumors, regeneration of blood flow, in situ $\mathrm{O}_{2}$ generation by decomposing $\mathrm{H}_{2} \mathrm{O}_{2}$, or microvascular alteration), ii) strategies for working with low oxygen concentrations (reduced $\mathrm{O}_{2}$ consumption or type I-triggered reaction), and iii) strategies combined PDT with hypoxia-activated therapies (chemotherapy, photothermal therapy (PTT), or immunotherapy). Based on these classifications, we introduce updated research centering on recently published papers without overlap with previous reports.

Han et al. $^{93}$ demonstrated the use of hyperthermiaresponsive micelles containing near-infrared (NIR)responsive PSs, which simultaneously induced thermal cycloreversion for PTT and singlet-oxygen generation for PDT without the participation of tumor oxygen. The small size $(\sim 50 \mathrm{~nm})$, positively charged surface $(\sim 4 \mathrm{mV})$, and tumor $\mathrm{pH}$ (6.8)-responsive property of the micelles were expected to enhance their cellular binding and tissue penetration, which improved their therapeutic efficiency against multicellular spheroids of 4T1 cancer cells, as well as in a 4T1-xenograft mouse model. This study showed an improved result of oxygen-independent PDT combined with PTT toward hypoxic tumors under mild NIR irradiation. Luo et al. described how hybrid protein oxygen carriers were conjugated with doxorubicin (an anticancer drug) and chlorin e6 (a chemical PS) via the disulfide linkage of hemoglobin and human serum albumin ${ }^{94}$. Owing to its oxygen-carrying ability, the protein hybrid complex supplied oxygen to tumors via caveolae endothelial transcytosis. Concurrently, with the downregulated expression of HIF- $1 \alpha$, multidrug resistance gene 1 , and Pglycoprotein, enhanced effects of chemotherapy and PDT were observed. In a more recent study, Zhang and coworkers reported an oxygen self-supplying system using fluorinated photosensitizer-based block copolymers ${ }^{95}$. Owing to the high affinity of fluorine for oxygen, oxygen could be adsorbed into the hydrophobic core in amphiphilic block copolymers and simultaneously delivered to tumor sites. They demonstrated that polymeric NPs were effectively taken up by cancer cells via a $\mathrm{pH}$-responsive route in a weakly acidic tumor microenvironment. Zhao 


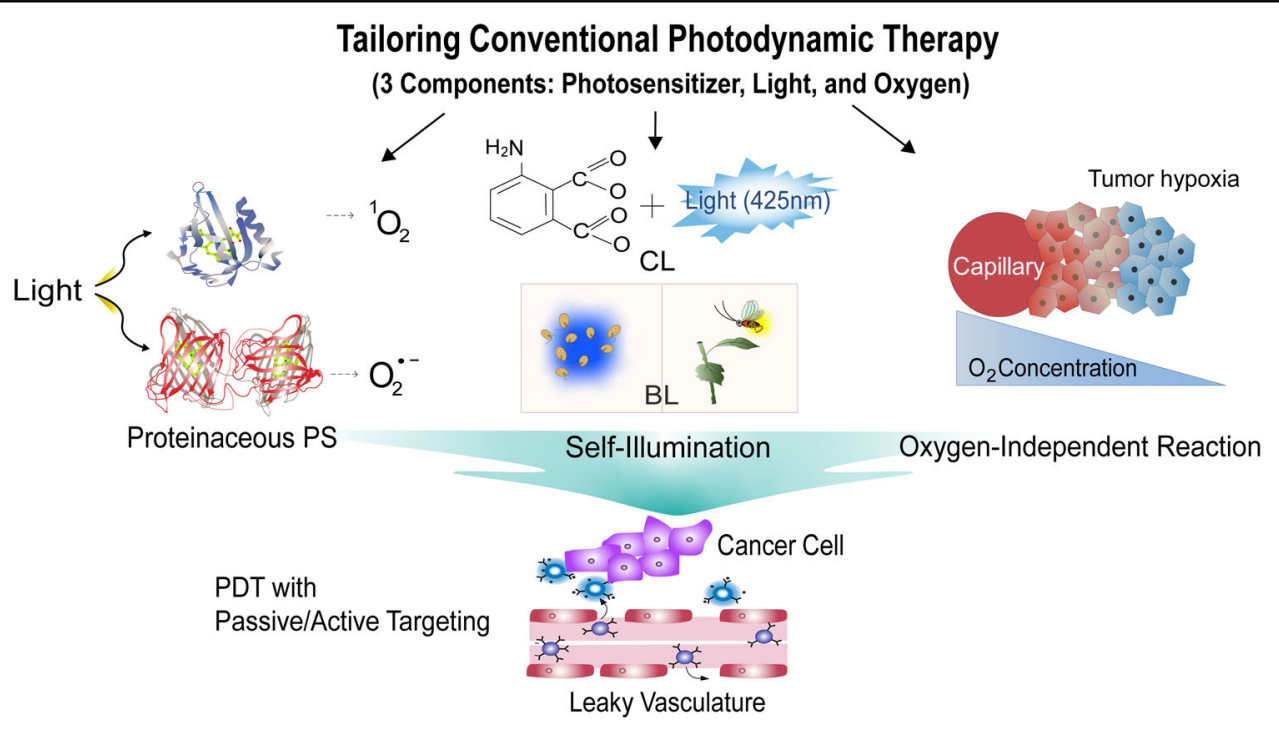

Fig. 2 Schematic overview of cancer treatment using smart PDT strategies to overcome the innate problems of conventional PDT. Three PDT approaches, including proteinaceous PS, self-illumination, and oxygen-independent methods are highlighted in this review. CL chemiluminescence, BL bioluminescence.

et al. developed a nanocomposite consisting of upconversion NPs (UCNPs) and a NIR light-triggered PS (iridium (III) complex), where an effective HIF- $1 \alpha$ inhibitor (YC-1) was physically adsorbed onto the hydrophobic layer at the surfaces of the UCNPs ${ }^{96}$. Since HIF- $1 \alpha$ is an attractive target for tumor therapy, and an iridium (III) complex with a long-lived triplet excited state can provide high singlet-oxygen generation, this nanocomposite showed antitumor effects on MDA-MB-231 tumorbearing mice even at low oxygen content, and permitted deep tissue light penetration.

Given the impact of hypoxia on resistance to tumor therapy, a specific oxygen supply or in situ oxygenation only around the tumor would be the best strategy to achieve the full potential of PDT in a hypoxic tumor. However, when a variety of oxygen generators, oxygen carriers, or nanoconjugates are employed in PDT, the oxygen toxicity in vivo should be carefully considered in future studies, as locally increased partial pressures of oxygen can lead to hyperoxia, which may be detrimental to the lungs and the central nervous system. Furthermore, type II reactions are considered the most common pathway in photodynamic reactions, but it should be mentioned that type III (triplet-doublet interaction) and type IV (photoisomerization) reactions can exert cytotoxic effects directly on intracellular structures without the mediation of oxygen ${ }^{97}$. Therefore, in addition to fundamental research on hypoxia, it is anticipated that the use of novel PS transporters or light-inducible self-regulating PS molecules will be an excellent alternative for the treatment of hypoxic tumors.

\section{Concluding remarks}

We discussed various strategies that have improved traditional PDT, focusing on three components of PDT (PS, light, and oxygen), as summarized in Fig. 2. To demonstrate the usefulness of PDT, which has been developed over its long history, extensive research on PDT should be conducted, including studies of safety, biodegradability, and tumor specificity by active targeting, along with examining targeting mechanisms, physicochemical factors, and the physiological characteristics of the tumor microenvironment. While the benchtop-tobedside translational approach for recently advanced PDTs is still a daunting process, these new insights brighten the clinical outlook for using PDT, thus ensuring next generation strategies. Although not covered in detail in this review, these PDTs can be combined with immunotherapy via immunomodulation, as recently reported elsewhere ${ }^{98-100}$. Therefore, more progressive approaches in conjunction with existing methods or new technologies will promote PDT development for targeted cancer treatment.

In conclusion, we highlighted recent advances in PDT for effective tumor treatment. The key aspect in PDT is ROS generation by light-activated PSs, which leads to tumor and vasculature killing via different cell death pathways and/or immune response activation. However, despite its clinical potential, it is not easy to optimize the PS, light, and oxygen depletion factors of PDT in cancer therapy, primarily due to the complexity of the tumor microenvironment. Beyond the barriers of conventional PDTs, smart approaches in combination with novel bio/ 
nanomaterials or insights have been recently developed, which include the use of proteinaceous PSs, selfilluminating methods, and oxygen-independent strategies. These developed PDTs are necessary to ensure effective and selective delivery to tumor sites as well as in vivo safety, and they have contributed to the increasing usefulness of PDT in cancer treatment. In combination with new materials or other therapeutic modalities, it is anticipated that safer, more effective, and more biocompatible PDT will bring even greater advances in cancer therapy and molecular medicine.

\section{Acknowledgements}

This work was supported by the National Research Foundation (NRF) grant funded by the Korean government (MSIT) (No. 2019R1A2C2004858) and the Korea Institute of Science and Technology (KIST) Institutional Program (Project No. 2Z06270-20-137). This work was also supported by the Basic Science Research Program (No. 2012R1A6A1029029 and 2020R1A6A1A06046728) through the NRF funded by the Ministry of Education, Korea.

\begin{abstract}
Author details
'Department of Life Science, Hanyang University, Seoul 04763, Republic of Korea. ${ }^{2}$ Department of HY-KIST Bio-Convergence, Hanyang University, Seoul 04763, Republic of Korea. ${ }^{3}$ Research Institute for Natural Sciences, Hanyang University, Seoul 04763, Republic of Korea. ${ }^{4}$ Research Institute for Convergence of Basic Sciences, Hanyang University, Seoul 04763, Republic of Korea. ${ }^{5}$ Institute of Nano Science and Technology, Hanyang University, Seoul 04763, Republic of Korea
\end{abstract}

\section{Conflict of interest}

The authors declare no competing interests.

\section{Publisher's note}

Springer Nature remains neutral with regard to jurisdictional claims in published maps and institutional affiliations.

Received: 5 February 2021 Revised: 4 March 2021 Accepted: 8 March 2021. Published online: 8 April 2021

\section{References}

1. Kelly, J. F. \& Snell, M. E. Hematoporphyrin derivative-possible aid in diagnosis and therapy of carcinoma of bladder. J. Urol. 115, 150-151 (1976).

2. Dolmans, D. E. J. G. J., Fukumura, D. \& Jain, R. K. Photodynamic therapy for cancer. Nat. Rev. Cancer 3, 380-387 (2003).

3. Juarranz, A., Jaen, P., Sanz-Rodriguez, F., Cuevas, J. \& Gonzalez, S. Photodynamic therapy of cancer. Basic principles and applications. Clin. Transl. Oncol. 10, 148-154 (2008).

4. Vrouenraets, M. B., Visser, G. W. M., Snow, G. B. \& van Dongen, G. A. M. S. Basic principles, applications in oncology and improved selectivity of photodynamic therapy. Anticancer Res. 23, 505-522 (2003).

5. Castano, A. P., Mroz, P. \& Hamblin, M. R. Photodynamic therapy and antitumour immunity. Nat. Rev. Cancer 6, 535-545 (2006).

6. Gollnick, S. O. \& Brackett, C. M. Enhancement of anti-tumor immunity by photodynamic therapy. Immunol. Res. 46, 216-226 (2010).

7. Yu, X. H. et al. Inhibiting metastasis and preventing tumor relapse by triggering host immunity with tumor-targeted photodynamic therapy using photosensitizer-loaded functional nanographenes. ACS Nano. 11, 10147-10158 (2017).

8. Li, W. et al. Targeting photodynamic and photothermal therapy to the endoplasmic reticulum enhances immunogenic cancer cell death. Nat. Commun. 10, https://doi.org/10.1038/s41467-019-11269-8 (2019).

9. Foote, C. S. Definition of type-I and type-II photosensitized oxidation. Photochem. Photobiol. 54, 659-659 (1991).

10. Schmidt, R. Photosensitized generation of singlet oxygen. Photochem. Photobiol. 82, 1161-1177 (2006).
11. Almeida, R. D., Manadas, B. J., Carvalho, A. P. \& Duarte, C. B. Intracellular signaling mechanisms in photodynamic therapy. Bba-Rev. Cancer 1704, 59-86 (2004).

12. Mroz, P., Yaroslavsky, A., Kharkwal, G. B. \& Hamblin, M. R. Cell death pathways in photodynamic therapy of cancer. Cancers 3, 2516-2539 (2011).

13. Oleinick, N. L. \& Evans, H. H. The photobiology of photodynamic therapy: cellular targets and mechanisms. Radiat. Res. 150, S146-S156 (1998).

14. Oleinick, N. L., Morris, R. L. \& Belichenko, I. The role of apoptosis in response to photodynamic therapy: what, where, why, and how. Photochem. Photobiol. Sci. 1, 1-21 (2002).

15. Bonora, M. \& Pinton, P. The mitochondrial permeability transition pore and cancer: molecular mechanisms involved in cell death. Front. Oncol. 4, 302 (2014).

16. Moserova, I. \& Kralova, J. Role of ER stress response in photodynamic therapy: ROS generated in different subcellular compartments trigger diverse cell death pathways. PLoS ONE 7, e32972 (2012).

17. Hitomi, J. et al. Involvement of caspase-4 in endoplasmic reticulum stressinduced apoptosis and A $\beta$-induced cell death. J. Cell Biol. 165, 347-356 (2004).

18. Lin, C.-W., Shulok, J. R., Kirley, S. D., Cincotta, L. \& Foley, J. W. Lysosomal localization and mechanism of uptake of Nile blue photosensitizers in tumor cells. Cancer Res. 51, 2710-2719 (1991).

19. Lin, C. W. et al. Photodynamic destruction of lysosomes mediated by Nile blue photosensitizers. Photochem. Photobiol. 58, 81-91 (1993).

20. Coupienne, I., Fettweis, G., Rubio, N., Agostinis, P. \& Piette, J. 5-ALA-PDT induces RIP3-dependent necrosis in glioblastoma. Photochem. Photobiol. Sci. 10, 1868-1878 (2011).

21. Miki, Y., Akimoto, J., Moritake, K., Hironaka, C. \& Fujiwara, Y. Photodynamic therapy using talaporfin sodium induces concentration-dependent programmed necroptosis in human glioblastoma T98G cells. Lasers Med. Sci. 30, 1739-1745 (2015).

22. Liang, L. M., Bi, W. X.\& Tian, Y. Y. Autophagy in photodynamic therapy. Trop. J. Pharm. Res. 15, 885-889 (2016).

23. Song, C. F. et al. Photodynamic therapy induces autophagy-mediated cell death in human colorectal cancer cells via activation of the ROS/JNK signaling pathway. Cell Death Dis. 11, https://doi.org/10.1038/s41419-02003136-y (2020).

24. Mroz, P., Yaroslavsky, A., Kharkwal, G. B. \& Hamblin, M. R. Cell death pathways in photodynamic therapy of cancer. Cancers 3, 2516-2539 (2011).

25. Yoo, J. O. \& Ha, K. S. New insights into the mechanisms for photodynamic therapy-induced cancer cell death. Int. Rev. Cel. Mol. Biol. 295, 139-174 (2012).

26. Kessel, D. \& Oleinick, N. L. Cell death pathways associated with photodynamic therapy: an update. Photochem. Photobiol. 94, 213-218 (2018).

27. Masoud, G. N. \& Li, W. HIF-1 alpha pathway: role, regulation and intervention for cancer therapy. Acta Pharm. Sin. B 5, 378-389 (2015).

28. Borgia, F. et al. Early and late onset side effects of photodynamic therapy. Biomedicines 6, https://doi.org/10.3390/biomedicines6010012 (2018).

29. Sylantiev, C., Schoenfeld, N., Mamet, R., Groozman, G. B. \& Drory, V. E. Acute neuropathy mimicking porphyria induced by aminolevulinic acid during photodynamic therapy. Muscle Nerve 31, 390-393 (2005).

30. Frangioni, J. V. In vivo near-infrared fluorescence imaging. Curr. Opin. Chem. Biol. 7, 626-634 (2003).

31. Freitas, I. Facing hypoxia-a must for photodynamic therapy. J. Photochem Photobiol. B 2, 281-282 (1988).

32. Lucky, S. S., Soo, K. C. \& Zhang, Y. Nanoparticles in photodynamic therapy. Chem. Rev. 115, 1990-2042 (2015).

33. Pass, H. I. Photodynamic therapy in oncology-mechanisms and clinical use. J. Natl Cancer Inst. 85, 443-456 (1993).

34. Kwiatkowski, S. et al. Photodynamic therapy-mechanisms, photosensitizers and combinations. Biomed. Pharmacother. 106, 1098-1107 (2018).

35. Agostinis, P. et al. Photodynamic therapy of cancer: an update. CA Cancer J. Clin. 61, 250-281 (2011).

36. Ethirajan, M., Chen, Y. H., Joshi, P. \& Pandey, R. K. The role of porphyrin chemistry in tumor imaging and photodynamic therapy. Chem. Soc. Rev. 40, 340-362 (2011).

37. Mfouo-Tynga, I. S., Dias, L. D., Inada, N. M. \& Kurachi, C. Biophysical and biological features of third generation photosensitizers used in anticancer photodynamic therapy: review. Photodiagn. Photodyn. 34, https://doi.org/ 10.1016/j.pdpdt.2020.102091 (2020). 
38. Polo, L., Valduga, G., Jori, G. \& Reddi, E. Low-density lipoprotein receptors in the uptake of tumour photosensitizers by human and rat transformed fibroblasts. Int. J. Biochem. Cell Biol. 34, 10-23 (2002).

39. Jeong, $\mathrm{H}$. et al. Photosensitizer-conjugated human serum albumin nanoparticles for effective photodynamic therapy. Theranostics 1, 230-239 (2011).

40. Sandland, J. \& Boyle, R. W. Photosensitizer antibody-drug conjugates: past, present, and future. Bioconjugate Chem. 30, 975-993 (2019).

41. Shu, X. et al. A genetically encoded tag for correlated light and electron microscopy of intact cells, tissues, and organisms. PLoS Biol. 9, https://doi.org/ 10.1371/journal.pbio.1001041 (2011).

42. Kim, M. et al. Colorimetric determination of singlet oxygen scavengers using a protein photosensitizer. Biochip J. 14, 148-157 (2020).

43. Westberg, M., Holmegaard, L., Pimenta, F. M., Etzerodt, M. \& Ogilby, P. R. Rational design of an efficient, genetically encodable, protein-encased singlet oxygen photosensitizer. J. Am. Chem. Soc. 137, 1632-1642 (2015).

44. Westberg, M., Bregnhoj, M., Etzerodt, M. \& Ogilby, P. R. No photon wasted: an efficient and selective singlet oxygen photosensitizing protein. J. Phys. Chem. B 121, 9366-9371 (2017).

45. Torra, J. et al. Singlet oxygen photosensitisation by the fluorescent protein Pp2FbFP L30M, a novel derivative of Pseudomonas putida flavin-binding Pp2FbFP. Photochem. Photobiol. Sci. 14, 280-287 (2015).

46. Bulina, M. E. et al. A genetically encoded photosensitizer. Nat. Biotechnol. 24, 95-99 (2006).

47. Carpentier, P., Violot, S., Blanchoin, L. \& Bourgeois, D. Structural basis for the phototoxicity of the fluorescent protein KillerRed. Febs. Lett. 583, 2839-2842 (2009).

48. Roy, A., Carpentier, P., Bourgeois, D. \& Field, M. Diffusion pathways of oxygen species in the phototoxic fluorescent protein KillerRed. Photochem. Photobiol. Sci. 9, 1342-1350 (2010).

49. Takemoto, K. et al. SuperNova, a monomeric photosensitizing fluorescent protein for chromophore-assisted light inactivation. Sci. Rep. 3, 2629 (2013).

50. Sarkisyan, K. S. et al. KillerOrange, a genetically encoded photosensitizer activated by blue and green light. PLOS ONE 10, e0145287 (2015).

51. Riani, Y. D., Matsuda, T., Takemoto, K. \& Nagai, T. Green monomeric photosensitizing fluorescent protein for photo-inducible protein inactivation and cell ablation. BMC Biol. 16, https://doi.org/10.1186/s12915-018-0514-7 (2018).

52. Shcherbo, D. et al. Far-red fluorescent tags for protein imaging in living tissues. Biochem. J. 418, 567-574 (2009).

53. Trewin, A. J. et al. Light-induced oxidant production by fluorescent proteins. Free. Radic. Biol. Med. 128, 157-164 (2018).

54. Mikkelsen, R. B. \& Wardman, P. Biological chemistry of reactive oxygen and nitrogen and radiation-induced signal transduction mechanisms. Oncogene 22, 5734-5754 (2003)

55. Moan, J. \& Berg, K. The photodegradation of porphyrins in cells can be used to estimate the lifetime of singlet oxygen. Photochem. Photobiol. 53, 549-553 (1991).

56. Nowis, D. et al. Direct tumor damage mechanisms of photodynamic therapy. Acta Biochim. Pol. 52, 339-352 (2005).

57. Bulina, M. E. et al. A genetically encoded photosensitizer. Nat. Biotechnol. 24, 95-99 (2006).

58. Liao, Z. X., Li, Y. C., Lu, H. M. \& Sung, H. W. A genetically-encoded KillerRed protein as an intrinsically generated photosensitizer for photodynamic therapy. Biomaterials 35, 500-508 (2014).

59. Ryumina, A. P. et al. Flavoprotein miniSOG as a genetically encoded photosensitizer for cancer cells. Biochem. Biophys. Acta 1830, 5059-5067 (2013).

60. Kim, E. H. et al. Self-luminescent photodynamic therapy using breast cancer targeted proteins. Sci. Adv. 6, https://doi.org/10.1126/sciadv.aba3009 (2020).

61. Jay, D. G. Selective destruction of protein function by chromophore-assisted laser inactivation. Proc. Natl Acad. Sci. USA 85, 5454-5458 (1988).

62. Bulina, M. E. et al. Chromophore-assisted light inactivation (CALI) using the phototoxic fluorescent protein KillerRed. Nat. Protoc. 1, 947-953 (2006).

63. Stolik, S., Delgado, J. A., Perez, A. \& Anasagasti, L. Measurement of the penetration depths of red and near infrared light in human "ex vivo" tissues. J. Photochem. Photobiol. B 57, 90-93 (2000).

64. Lovell, J. F., Liu, T. W. B., Chen, J. \& Zheng, G. Activatable photosensitizers for imaging and therapy. Chem. Rev. 110, 2839-2857 (2010).

65. Magalhaes, C. M., da Silva, J. C. G. E. \& da Silva, L. P. Chemiluminescence and bioluminescence as an excitation source in the photodynamic therapy of cancer: a critical review. Chemphyschem 17, 2286-2294 (2016).
66. Nogami, T., Hashimoto, M. \& Tsukagoshi, K. Metal ion analysis using microchip CE with chemiluminescence detection based on 1,10-phenanthrolinehydrogen peroxide reaction. J. Sep. Sci. 32, 408-412 (2009).

67. Heller, C. A., Carlisle, D. T. \& Henry, R. A. Chemiluminescence: Quantum yields for luminol and a biisoquinolinium salt. J. Lumin. 4, 81-88 (1971).

68. Chen, G. et al. A review of enhancers for chemiluminescence enzyme immunoassay. Food Agric. Immunol. 28, 315-327 (2017).

69. Yuan, H. X. et al. Chemical molecule-induced light-activated system for anticancer and antifungal activities. J. Am. Chem. Soc. 134, https://doi.org/ 10.1021/ja304986t (2012).

70. Zhang, Y. R. et al. Small molecule-initiated light-activated semiconducting polymer dots: an integrated nanoplatform for targeted photodynamic therapy and imaging of cancer cells. Anal. Chem. 86, 3092-3099 (2014).

71. Mao, D. et al. Chemiluminescence-guided cancer therapy using a chemiexcited photosensitizer. Chem 3, 991-1007 (2017).

72. Xu, X. Q. et al. A self-illuminating nanoparticle for inflammation imaging and cancer therapy. Sci. Adv. 5, https://doi.org/10.1126/sciadv.aat2953 (2019).

73. Yang, K. et al. Self-illuminating photodynamic therapy with enhanced therapeutic effect by optimization of the chemiluminescence resonance energy transfer step to the photosensitizer. Bioconjug. Chem. 31, 595-604 (2020).

74. Wilson, T. \& Hastings, J. W. Bioluminescence. Annu. Rev. Cell Dev. Biol. 14, 197-230 (1998).

75. Haddock, S. H. D., Moline, M. A. \& Case, J. F. Bioluminescence in the Sea. Annu. Rev. Mar. Sci. 2, 443-493 (2010).

76. Contag, C. H. \& Bachmann, M. H. Advances in vivo bioluminescence imaging of gene expression. Annu. Rev. Biomed. Eng. 4, 235-260 (2002).

77. Lim, J. H. et al. Surface-tunable bioluminescence resonance energy transfer via geometry-controlled ZnO nanorod coordination. Small 11, 3469-3475 (2015).

78. Nguyen, D. L. et al. Detection of matrix metalloproteinase activity by bioluminescence via intein-mediated biotinylation of luciferase. Sensors $\mathbf{1 8}$ 875-884 (2018).

79. Theodossiou, T. et al. Firefly luciferin-activated Rose Bengal: In vitro photodynamic therapy by intracellular chemiluminescence in transgenic $\mathrm{NIH}$ 3T3 cells. Cancer Res. 63, 1818-1821 (2003).

80. Hsu, C. Y., Chen, C. W., Yu, H. P., Lin, Y. F. \& Lai, P. S. Bioluminescence resonance energy transfer using luciferase-immobilized quantum dots for self-illuminated photodynamic therapy. Biomaterials 34, 1204-1212 (2013).

81. Kim, Y. R. et al. Bioluminescence-activated deep-tissue photodynamic therapy of cancer. Theranostics 5, 805-817 (2015).

82. Yang, $Y$. et al. Biodegradable polymer nanoparticles for photodynamic therapy by bioluminescence resonance energy transfer. Biomacromolecules 19, 201-208 (2018).

83. Al-Ani, A. W. et al. Listeria innocua Dps as a nanoplatform for bioluminescence based photodynamic therapy utilizing Gaussia princeps luciferase and zinc protoporphyrin IX. Nanomedicine 20, 102005 (2019).

84. Shramova, E. I., Proshkina, G. M., Chumakov, S. P., Khodarovich, Y. M. \& Deyev, S. M. Flavoprotein miniSOG cytotoxisity can be induced by bioluminescence resonance energy transfer. Acta Nat. 8, 118-123 (2016).

85. Henderson, B. W. \& Fingar, V. H. Relationship of tumor hypoxia and response to photodynamic treatment in an experimental mouse-tumor. Cancer Res. 47, 3110-3114 (1987).

86. Pouyssegur, J., Dayan, F. \& Mazure, N. M. Hypoxia signalling in cancer and approaches to enforce tumour regression. Nature 441, 437-443 (2006)

87. Huang, Z. et al. Photodynamic therapy for treatment of solid tumorsPotential and technical challenges. Technol. Cancer Res. Treat. 7, 309-320 (2008).

88. Du, B. J. \& Tung, C. H. Enzyme-assisted photodynamic therapy based on nanomaterials. ACS Biomater. Sci. Eng. 6, 2506-2517 (2020).

89. Mitra, S. et al. Photodynamic therapy mediates the oxygen-independent activation of hypoxia-inducible factor 1 alpha. Mol. Cancer Ther. 5, 3268-3274 (2006).

90. Weijer, R. et al. Inhibition of hypoxia inducible factor 1 and topoisomerase with acriflavine sensitizes perihilar cholangiocarcinomas to photodynamic therapy. Oncotarget 7, 3331-3346 (2016).

91. Broekgaarden, M. et al. Inhibition of hypoxia-inducible factor 1 with acriflavine sensitizes hypoxic tumor cells to photodynamic therapy with zinc phthalocyanine-encapsulating cationic liposomes. Nano Res. 9, 1639-1662 (2016). 
92. Li, X. S., Kwon, N., Guo, T., Liu, Z. \& Yoon, J. Innovative strategies for hypoxictumor photodynamic therapy. Angew. Chem. Int. Ed. 57, 11522-11531 (2018).

93. Han, Y. et al. Oxygen-independent combined photothermal/photodynamic therapy delivered by tumor acidity-responsive polymeric micelles. J. Control Release 284, 15-25 (2018).

94. Luo, Z. Y. et al. Tumor-targeted hybrid protein oxygen carrier to simultaneously enhance hypoxia-dampened chemotherapy and photodynamic therapy at a single dose. Theranostics 8, 3584-3596 (2018).

95. Liu, Z. Y. et al. Sensitization of hypoxic tumor to photodynamic therapy via oxygen self-supply of fluorinated photosensitizers. Biomacromolecules 20 , 4563-4573 (2019).

96. Zhao, J., Sun, S., Li, X., Zhang, W. \& Gou, S. Enhancing photodynamic therapy efficacy of upconversion-based nanoparticles conjugated with a long-lived triplet excited state iridium(III)-naphthalimide complex: toward highly enhanced hypoxia-inducible factor-1. ACS Appl. Bio Mater. 3, 252-262 (2020).

97. Scherer, K. M., Bisby, R. H., Botchway, S. W. \& Parker, A. W. New approaches to photodynamic therapy from types I, II and III to type IV using one or more photons. Anticancer Agents Med. Chem. 17, 171-189 (2017).

98. Song, W. et al. Enhanced immunotherapy based on photodynamic therapy for both primary and lung metastasis tumor eradication. ACS Nano 12, 1978-1989 (2018).

99. Shen, L. J. et al. Recent progress in tumor photodynamic immunotherapy. Chin. Chem. Lett. 31, 1709-1716 (2020).

100. Wang, $M$. et al. Cancer photo-immunotherapy: from bench to bedside Theranostics 11, 2218-2231 (2021). 